\title{
Characteristics and Distribution of Microplastics in Shoreline Sediments of the Yangtze River, Main Tributaries and Lakes in China-From Upper Reaches to the Estuary
}

\author{
Wenfeng Li ( $\sim$ liwf86@ms.xjb.ac.cn ) \\ Xinjiang Institute of Ecology and Geography \\ Jia Duo \\ Xinjiang Institute of Ecology and Geography \\ Rehemanjiang Wufuer \\ Xinjiang Institute of Ecology and Geography \\ Shuzhi Wang \\ Xinjiang Institute of Ecology and Geography \\ Xiangliang Pan \\ Zhejiang University of Technology
}

\section{Research Article}

Keywords: microplastics, sediment, characteristics, distribution, Yangtze River.

Posted Date: June 1st, 2021

DOI: https://doi.org/10.21203/rs.3.rs-504372/v1

License: (c) (i) This work is licensed under a Creative Commons Attribution 4.0 International License. Read Full License

Version of Record: A version of this preprint was published at Environmental Science and Pollution Research on February 22nd, 2022. See the published version at https://doi.org/10.1007/s11356-02118284-7. 


\section{Abstract}

Microplastics (MPs) pervade the environment and increasingly threaten both natural ecosystems and human health. In this study, we investigated MP particle concentrations in sediment samples collected from 54 sites along the banks of the Yangtze River and its major tributaries and on lakeshores. The main polymer types found in the samples were polypropylene (PP), polystyrene (PS), and polyethylene (PE). MP particle abundance in the various types of locations was 35-51968 particles/kg dry weight (d.w.) on the banks of the main river, 52-1463 particles/kg (d.w.) on the banks of tributaries and 2574-23685 particles $/ \mathrm{kg}$ (d.w.) on lakeshores. Correlation between MP abundance and mean annual runoff of each upstream tributary was significant, which suggests that increased runoff brings more microplastic waste to streambank sediments. The most common shape of MP particles in all upstream samples was flake and in downstream samples it was foam. Small microplastic particles $(<0.050 \mathrm{~mm})$ were predominant at all sites in this study and the minimum particle size in samples from the Yangtze river banks was 0.065 $\mathrm{mm}$. Average abundance of MP particles on the shores of the source lake was 9069 particles $/ \mathrm{kg}$ around the inlet but only 866 particles $/ \mathrm{kg}$ around the outlet; the difference was due to interception associated with sedimentation and precipitation in the lake. This study provides data and presents a theoretical basis for the analysis of the accumulation and distribution of MPs in sediment in the Yangtze River basin.

\section{Introduction}

Plastics are extremely versatile manufactured materials. China produced $102.3 \mathrm{Mt}$ plastic materials in 2017, the European Union (EU) 64.4 Mt and the North American Free Trade Agreement (NAFTA) countries 61.6 Mt (Plastics Europe, 2018). Microplastic (MP) particles are plastics $<5 \mathrm{~mm}$ in size that originate from primary and secondary MPs (Endo et al., 2005; Ryan et al., 2009). MP pollution is a global environmental concern because of the ubiquity of MPs in the environment and the risks they pose to ecosystems (do Sul et al., 2014; Rachman et al., 2018). In general, small MPs cause most harm to the natural environment because they are easily available to higher species and are concentrated as they move up the biological food chain (Canesi et al., 2015). MPs can also become vectors of toxic and harmful substances that can cause food or water pollution (Zhang et al., 2018).

Most research into MPs has focused on its marine distribution, but there has been a recent increase in research into the sources, distribution, abundance and biological effects of MPs (Hidalgo et al., 2012; Woodall et al., 2014; Desforges et al., 2014; Zhou et al., 2018; Ivar do Sul et al., 2014; Nguyen et al., 2019). The degree of freshwater MP pollution is comparable to or more serious than oceanic pollution (Wu et al., 2018). It has been estimated that globally 4.8-12.7 Mt plastic debris from inland water systems and terrestrial ecosystems has been transported to the ocean by riverine transport in 2010 (Jambeck et al., 2015), and that 262.3-270.2 Mt plastic wastes remains in land-based environments, such as surface waters, and bottom and shoreline river and lake sediments and so on. Along a short length of ocean, there can be MPs from many sources, as there can be along a river, from its headwaters to the estuary, particularly if nearby cities are hotspots of MP pollution (Lebreton et al., 2017; Siegfried et al., 2017; Xu et 
al., 2018; Hu et al., 2018; He et al., 2021). There is little available literature describing research into MPs in freshwater environments, but what limited information there is shows extensive MP pollution in rivers, lakes, and reservoirs (Zhang et al., 2015; Peng et al., 2018; Wang et al., 2018).

In this study, we collected microplastic samples from the banks of the upper, middle and lower reaches of the Yangtze River, its main tributaries and the shores of lakes to estimate the distribution of MPs. The purpose of this study was to increase our knowledge of MP pollution in the riverbank sediment of China's largest river, its seven major tributaries and four freshwater lakes. We used laboratory tests of samples collected in field investigations to identify the probable sources of MP pollutants and to identify the shapes, polymer types, abundance, particle sizes, and distribution of MP particles in the Yangtze River basin.

\section{Materials And Methods}

\subsection{Study area}

The Yangtze River is the longest river in China. The main river flows through 11 provinces, regions and cities, and the basin covers about 2.05 million $\mathrm{km}^{2}$. The major tributaries of the upper Yangtze River, the Jinsha, Yalong, Minjiang, Tuojiang, Jialing, Wujiang and Hanjiang rivers, are abundant water resources that are also extremely important hydropower sources in China. By 2013, there were 80 large reservoirs and 381 medium-sized reservoirs in the upper reaches of the Yangtze River (Yao et al., 2016). There are about 760 lakes in the lower reaches of the Yangtze River with a total area of $17093 \mathrm{~km}^{2}$, the major lakes are Dongting Lake, Poyang Lake, Chaohu Lake and Taihu Lake. These lakes have accelerated the development of local agriculture, industry, shipping, aquaculture and tourism (Dong et al., 2013). A map and coordinates are shown in Fig. 1 and Table S1.

\subsection{Sampling}

A total of 162 sediment samples were taken from 54 selected sites on the Yangtze River banks, the banks of the main tributaries and the shores of some of the lakes, during May and June 2018. The distance over which samples were taken runs from Yushu, Qinghai province to the river estuary at Shanghai, along > $6000 \mathrm{~km}$ of the Yangtze river valley. The sampling site locations are shown in Fig. 1. Approximately $4 \mathrm{~kg}$ shoreline sediment samples were taken from a $1 \mathrm{~m} \times 1 \mathrm{~m}$ square at each site by lifting the top $2 \mathrm{~cm}$ of sediment using a stainless steel shovel (Murray et al. 2011). Every sample was taken randomly at each site using a mixed multipoint method. One square was chosen randomly for sampling and then 3 squares were chosen at $20 \mathrm{~m}$ intervals along an S-shaped route. The sediment specimens were mixed together in a fabric bag. The samples were sealed and stored in the laboratory at room temperature until required for analysis. Wet sediment samples were air-dried in the laboratory to avoid pollution of the MPs in the sample. MP particle abundance was determined based on the dry weight of the sediment sample.

\subsection{Sample analysis}


Each sample was homogenized in the laboratory without cross-contamination. About $500 \mathrm{~g}$ of each sample was sieved in a stainless steel sieve (300 mesh, $\Phi=0.050 \mathrm{~mm}$ ) and leached using pressurized water until the leaching water ran clear. Sieve residue was collected in a volumetric flask that had been cleaned using ultrapure water and then, for flotation of microplastic particles, mixed and shaken with an $\mathrm{NaCl}$-saturated solution $\left(1.2 \mathrm{~g} / \mathrm{cm}^{3}\right)$ three times and once with a $\mathrm{ZnCl}_{2}$-saturated solution $\left(1.6 \mathrm{~g} / \mathrm{cm}^{3}\right)$. The supernatant of each flotation was decanted into a $500 \mathrm{~mL}$ glass beaker and vacuum filtered with 10-20 $\mu \mathrm{m}$ quantitative paper ( $\Phi=9 \mathrm{~cm}$, Xinxing, China). The filter membrane was washed with deionized filtered water, and the residue was carefully transferred to a clean Petri dish using a washed metallic needle before being covered and dried in a drying oven at $60^{\circ} \mathrm{C}$ for $>12 \mathrm{~h}$. Organic matter in the MPs was digested using methods described in (Li et al., 2020). Particles were observed using a microscope (PDOK 10X, Germany) with a multifunctional magnifying lamp and microscope (Olympas, IX81). Particles that were visually identified as or suspected to be plastics were transferred from the residues onto clean, black, smooth cardboard and classified according to shape (Nor et al., 2014; Zhou et al., 2018). All particles transferred to the cardboard were photographed using a digital camera (Nikon D3200, Japan) for particle counting and size measurement.

\subsection{Data analysis}

Smaller MP particles may not be classifiable due to their small size making their morphology indiscernible. Two or three typical larger particles were selected for polymer identification using attenuated total reflection with Fourier transform infrared spectroscopy (ATR-FTIR). ATR-FTIR spectra were recorded on a Bruker Tensor 27 FTIR spectroscope with Pike Miracle ATR accessories. ArcGIS10.2 was used to map the spatial distribution of MP particles. Nano Measurer 1.2 was used to size and count particles. WPS Office 2019 and OriginPro 8.5 (OriginLab Corporation, Northampton, MA) were used for data analysis. SPSS 17.0 was used for analysis of variance. All results concerning MP particle abundances are presented as the number of microplastic particles per dry mass of sediment (particles/kg).

\subsection{Quality assurance and quality control}

MP particles were separated and enumerated in different clean rooms. All containers and beakers were rinsed three times with filtered water at each individual step to avoid pollution. The samples were kept in closed spaces to reduce pollution from airborne MPs. All materials and vessels were covered with aluminum foil after each individual step. Cotton lab coats were worn at all times during analysis. Controls were produced using the same processing methods as those applied to the field samples and laboratory processes.

\section{Results}

\subsection{Shape categories, polymer type and distribution of MPs}


The shape categories used for MP particles were pellet, foam, fragment, flake, film, fiber and sponge (Zhou et al. 2018), as shown in Fig. 2. Pellet was hard, regular, disc-shaped, oval or cylindrical plastic particles. Foam was white expanded polystyrene particles. Film was thin and soft particles of plastic sheet. Fiber was long, curly remnants of dust screen or fishing nets. Fragment was irregularly shaped hard plastic debris. Flake was flat particles of various plastic woven bags. Sponge was particles that were yellow, porous and irregular.

The composition and classification of sediment samples were determined by attenuated total reflectance-Fourier transform infrared (ATR-FTIR) spectrometry using the detected spectra, as shown in Fig. 3. The FTIR characteristic absorbance bands of polyethylene (PE) are $1162 \mathrm{~cm}^{-1}$ and $720 \mathrm{~cm}^{-1}$, and of polypropylene (PP), 973-971 $\mathrm{cm}^{-1}$ and $156 \mathrm{~cm}^{-1}$. We found that the most plentiful polymers were in the categories film and pellet (PE), and fiber, flake and fragment (PP). We found that foam particles had distinct spectral bands for in-plane and out-of-plane bending vibrations of hydrogen in a benzene ring at wavenumbers $1070 \mathrm{~cm}^{-1}, 1028 \mathrm{~cm}^{-1}$ and $754 \mathrm{~cm}^{-1}$, and $700 \mathrm{~cm}^{-1}$, which are the characteristic peaks of polystyrene (PS). We also found sponge particles of polyurethane (PEU), as shown in Fig. $3(\mathbf{g})$.

Flake particles decreased from $46.3 \%$ of MPs in the upper reaches of the Yangtze River bank sediments to $10.2 \%$ in the lower reaches. In the middle reaches, the proportion of flake particles was $68.0 \%$; the greatest abundance was in the Wuhan section, $88.1 \%$. In contrast, foam particles were $4.9 \%$ in the upper reaches of the Yangtze and $59 \%$ in the lower reaches, a tenfold increase, as shown in Fig. 4(a). In the tributaries of the upper reaches of the Yangtze River, particles were primarily flake (maximum $76.1 \%$ ) and film (maximum $46.0 \%$ ), and the average proportion of foam particles was $<10 \%$. No spherical particles were found in the tributary sediments, as shown in Fig. 4(b). Particles representative of all seven shape categories were found in the lakeshore sediments: the average proportion of foam particles was $41.7 \%$, with a maximum of $64.4 \%$ for Poyang Lake; the average proportion of flake was $20.05 \%$ and of film, $13.8 \%$, as shown in Fig. 4(c).

\subsection{Abundance and size of MPs in the Yangtze River basin}

The abundance of MP particles in the Yangtze River banks is shown in Fig. 5(a). The abundance of MP particles in the beach sediments of the main urban basins along the Yangtze River varied greatly. The abundance of MP particles in riverbank sediments from Chongqing to Yueyang was relatively small, in the range 35-322 particles $/ \mathrm{kg}$. However, from Wuhan to Shanghai, the abundance of MP particles was relatively high, mainly in the range 278-51 968 particles $/ \mathrm{kg}$. The greatest abundance of MP particles in sediment samples was found in the Wuhan region, followed by the Jiujiang, Shanghai and Nanjing regions. The abundance of MP particles in the Yangtze estuary was 992 particles $/ \mathrm{kg}$. Abundance in the upstream tributaries was in the range 254-1463 particles/kg, as shown in Fig. 5(b); the highest abundance was found in the Mingjiang River bank. The abundance of MP particles in the shores of Dongting Lake (2576 particles/kg), Poyang Lake (8017 particles/kg), Chaohu Lake (5460 particles/kg) and Taihu Lake (23 685 particles $/ \mathrm{kg}$ ) were significantly greater than in the banks of the tributaries, as shown in Fig. 5(c). 
Microplastics were classified into six categories according to their size: $0-0.5 \mathrm{~mm}, 0.5-1.0 \mathrm{~mm}, 1.0-2.0$ $\mathrm{mm}, 2.0-3.0 \mathrm{~mm}, 3.0-4.0 \mathrm{~mm}$ and $4.0-5.0 \mathrm{~mm}$. Zhao et al. (2014) found that MPs of size $<0.5 \mathrm{~mm}$ were the most abundant MPs in the surface waters of the Yangtze River, which was attributed to long term degradation of small plastic debris. The size distributions of MPs in sediment determined by our experiments are shown in Fig. 6. The size range of MPs detected in the sediments of the main stream was $0.065 \pm 0.013-4.02 \pm 0.452 \mathrm{~mm}$, in tributaries $0.071 \pm 0.008-3.91 \pm 0.883 \mathrm{~mm}$, and in lakes $0.073 \pm$ $0.016-4.10 \pm 0.593 \mathrm{~mm}$ (Table S2). MPs $<0.5 \mathrm{~mm}$ were predominant in the sediments of the Yangtze River banks (average 66.7\%), tributary riverbanks (average 64.1\%) and lakeshores (average 65.9\%); the greatest proportions were $88.1 \%$ in the Wuhan region, $70 \%$ in Tuojiang River banks and $79.3 \%$ in Chaohu Lakeshores. The general trend was that larger MP particles ( $>2 \mathrm{~mm}$ ) were less abundant in the sediments of the Yangtze River banks (in the range 0.0-12.85\%), tributary riverbanks (in the range 3.9-7.5\%) and lakeshores (in the range 5.5-24.56\%). We found that sample sites with high abundance of flake (PP) and foam (PS) particles had higher proportions of small particles. Thin flake and expanded PS are more prone to weathering to form MP particles in the environment; after long term secondary weathering in shoreline sediments, they form smaller particles. Lower density particles of polymer PE, PP and expanded PS that are distributed on the surfaces of rivers and lakes are more likely to be trapped in the riverbank and shoreline sediments because they are influenced by tides and wind, thus increasing the proportion of small MPs in the sediments of riverbanks and lakeshores.

\subsection{Microplastics contamination in tributary riverbanks}

The distributions of MP particles showed different trends upstream and downstream in each tributary (Table S3 and Fig. S1). Flake particles were predominant in upstream samples, accounting for $73 \%$ on average. The greatest proportion of flake particles was $98.8 \%$ in the Mingjiang River samples. The proportion of fiber particles was $74.3 \%$ in upstream samples of the Tuojiang River. No pellet or sponge particles were found in the upstream samples. Downstream in the tributaries, film (average 39.2\%), flake (average 20.1\%), fragment (average 13.5\%) and foam (average 14.4\%) were the most common particle shapes found in the sediment samples. Fiber particles were found in the Jialing (41.8\%) and Wujiang (43.3\%) samples. Sponge particles were found only in the Jialing samples (3.6\%) and no pellet particles were found in any tributary samples. Abundance of MP particles in samples from the upper reaches of the tributaries were in the range 192-1004 particles/kg (average 418 particles/kg) and samples from the lower reaches were in the range 30-580 particles/kg (average 252 particles/kg) (Fig. 7a).

\subsection{Microplastic contamination characteristics of lakeshores}

Flake particles accounted for $32.6 \%$ on average, follow by fragment (24.2\%), foam and film accounted for $21.5 \%$ and $19.2 \%$, respectively, in the Dongting Lake samples. Foam, flake and fiber particles accounted respectively for $27.3 \%, 36.4 \%, 26.8 \%$ of the Chaohu Lake samples. Foam particles were predominant in samples from both Poyang Lake (58.4\%) and Taihu Lake (70.4\%). Foam particles were widespread; we found them in every lake sample, with abundance in the range $21.5-70.4 \%$. At the outlet of Dongting Lake, film and flake particles accounted for $90 \%$ of particles found in the samples. Foam particles 
accounted for $88.5 \%$ of the particles found in the Poyang Lake outlet samples. Foam, fragment, film, fiber and flake particles were predominant in both the Chaohu (81.7\%) and Taihu Lake outlet samples (82.9\%). Pellet and sponge particles had an abundance of $<10 \%$ in the inlet and outlet samples of these two lakes (Table S4 and Fig. S2). The abundance of MP particles differed significantly between inlet and outlet samples for all lakes, as shown in Fig. 7(b). The abundance of MP particles in lake inlet samples was in the range 2546-21 112 particles $/ \mathrm{kg}$ (average 9069 particles $/ \mathrm{kg}$ ); in lake outlet samples, abundance was in the range 30-2573 particles $/ \mathrm{kg}$ (average 866 particles $/ \mathrm{kg}$ ).

\subsection{Correlates of microplastics in the Yangtze River basin}

We found significant correlations between particle abundance in tributary samples and water runoff (Fig. 8a and Table S5). The abundance of flake particles along the Yangtze River banks was positively correlated with the total MP abundance in the main tributary (Fig. 8b and Table S6). There were significant correlations between the abundance of foam particles and total MP particle abundance in the main sampling areas of the Yangtze River (Fig. 8c and Table S7).

\section{Discussion}

\subsection{Source and distribution of MPs}

There may be a close relationship between human activities along the Yangtze River and the different types of MP particles found in our sampling (Wang et al., 2017; Yang et al., 2021). Different factors may contribute to the spatial differences observed in MP contaminants along the river. We frequently observed discarded foam floats and containers, plastic drums and bottles, fishing nets, woven plastic bags and food wrappers on the banks of the Yangtze River and its main tributaries and on the lakeshores during field sampling, all of which are potential sources of MP particles.

Flake particles predominated in the upstream Yangtze River samples. These may have originated from gradual damage to discarded plastic woven bags. Polystyrene foam particles were found mainly in the Yangtze River bank samples; these originated primarily from aquatic product packing boxes and discarded foam floats, as reported by Free et al. (2014) and Lee et al. (2015). Fiber particles were widely distributed in the Yangtze River bank samples, mainly due to the large use of wind fences in the economically less developed areas of the upper reaches of the Yangtze River, and to the fishery that has developed in the lower reaches of the river. Film particles originated mainly from the degradation of discarded plastic bags and agricultural mulch (Sintim et al., 2017). The main sources of fragment particles were discarded objects that resulted from human lifestyles and the industrial production of hard plastic products; over the long term, hard plastic products break and produce fragments (e.g., broken plastic tubs, buckets and bottles). Film and fragment particles were widely present in samples throughout the survey area, with a relatively high distribution in more populated areas that further illustrates the close relationship between MPs and human activities. Pellet particles were found in the middle and lower reaches of the Yangtze River bank samples, which we mainly attributed to improperly discarded industrially manufactured primary materials. More pellet particles were found in lakeshore sediments 
because a lake is an important destination for pellet particle migration in the drainage basin. Sponges are widely used in industry and commerce for shock absorption, packaging, and heat insulation, and they increase pollution by PEU MPs (Zhou et al., 2018).

\subsection{Microplastic contamination along the Yangtze River}

MP particle concentrations in different reaches of the Yangtze River were dissimilar because they were influenced by different environmental conditions and had different sources (Feng et al., 2021). Previous studies have suggested that MP concentrations in aquatic environments were mainly driven by population density or economic development (Lahens et al., 2018; Peng et al., 2018; Eriksen et al., 2013; Yonkos et al., 2014; Liu et al., 2019). We found that the abundance of MP particles along the Yangtze River banks was relatively high in major urban watersheds, but there was no significant correlation between abundance and local population size or economic development indicators. We conclude that MP particles in shoal sediments have a complex of sources, including local discarding of plastic products and continuous input from upstream rivers, and that local hydrological conditions maybe have an important effect on plastic deposition. The abundance of MPs in riverbank sediments is also a consequence of long term accumulation. Over time, different types of plastic products and MPs are weathered by various factors, a process that continuously increases MP particle abundance (Song et al., 2017).

In contrast to previous studies of MP accumulation, we found that the abundance of MPs in the sediments of the main Yangtze River basin is at an intermediate level (Ding et al., 2019; Lin et al., 2018; Klein et al., 2015). The Yangtze River has long been considered to be the largest source of MPs entering the ocean, but previous studies ignored the retention of MPs in shoal sediments, leading to overestimates of the flux of MPs from rivers into the ocean (Siegfried et al., 2017).

We found only particles of a single shape category and a low abundance of MP particles in the upper reaches of the Yangtze River. We attribute this to relatively little human disturbance in the area, thus reducing the number of possible sources and therefore the abundance of MP particles. In the middle and lower reaches of the river, the abundance of MP particles was greater. Greatest abundance was found around Wuhan, and the particles were mainly flakes. This may be related to local long term flood defenses and remediation. There were more foam and fiber particles in the lower reaches due to the developed fishery and transportation of aquatic products. The significant positive correlation between the abundance of foam particles and the total abundance of MP particles implies that PS plastic products are the main sources of MPs in the sediment samples of the main sampling areas of the Yangtze River. MP particle abundance was relatively high in the Yangtze estuary, which indicates that urban estuarine river input was an important source of MPs entering the ocean (Rech et al., 2014; Zhao et al., 2015). Tides may also be an important factor in the accumulation of MPs in estuaries (Xiong et al., 2015).

\subsection{Microplastics in the tributaries of the upper Yangtze River}


In the tributaries of the upper reaches of the Yangtze River, where human activities are less relevant, the abundance of MP particles is nonetheless relatively high. This is related to the types of polymer; polypropylene (PP) and polystyrene (PS) fragment easily in natural conditions (Song et al., 2017). The significant positive correlations between the abundance of flake particles and the total abundance of MP particles in tributary riverbank samples indicate that flake particles are predominant.

In the upper reaches of the tributaries, which are undeveloped, woven plastic bags and netted wind fencing discarded during the construction and maintenance of water conservancy and hydropower facilities produced a high abundance of MP particles. Particle shapes were mainly flake and fiber, and flake particles were predominant.

Agriculture and other human activity is more frequent in the lower reaches of each tributary, and both overall abundance and the number of shape categories in our samples increased. We found that flake particles decreased significantly in the lower reaches of each tributary. This observation suggests that a dam on the upper reaches of a tributary may have trapped considerable amounts of MPs that originated upstream of the dam (Zhang et al., 2015; Zhang et al., 2015; Xiong et al., 2019).

Runoff is believed to contribute to microplastic pollution (Gasperi et al., 2014; Dai et al., 2013). Significant correlation between MP particle abundance and mean annual runoff was found for each tributary of the upper Yangtze River. This suggests that increased runoff transports more surface microplastic waste into the rivers, and, as a result, the abundance of MP particles remaining in riverbank sediments may be increased by waves or wind. These particles will, over time, flow into the upper reaches of the Yangtze River and continue to be transported downstream, possibly into the oceanic system, thus becoming an important source of marine MPs.

\subsection{Characteristics of microplastics contamination in lakeshores}

The sediment MP particle concentrations found in lakeshore samples were high in comparison with some other studies, such as of the Caofeidian reclamation area (Zhou et al., 2016), Halifax Harbor in Nova Scotia, Canada (Mathalon et al., 2014), the Poyang Lake system (Jian et al., 2020), Dongting Lake (Wang et al., 2020) and Taihu Lake (Su et al., 2016).

Particles in all seven shape categories were found in all lakeshore sediment samples. Abundance of MP particles in lakeshore samples was significantly greater than in riverbank sediment samples because particles in rivers are mainly transported downstream, whereas particles in lakes are mainly deposited and thus remain in place. Abundances in the lake outlet samples were significantly less than those found at inlets. This is likely because the lake water outflow rate was low; still water provides good conditions for a large number of MP particles to be trapped in lake and lakeshore sediments (Xiong et al., 2019). Our findings imply that previous models may have overestimated the flux of MPs from land to ocean because they did not take into account the retention of MPs in river and lake systems. 


\section{Conclusions}

Our results show the ubiquity of MPs in sediments from the banks of the Yangtze River and tributaries and lakeshores. We also characterized the accumulation and distribution of MPs in the survey region. We identified seven shape categories across the entire Yangtze River basin and analyzed the main polymer types as polypropylene (PP), polystyrene (PS), and polyethylene (PE). The sources of the different categories of MPs were closely related to human activity along the Yangtze River. The higher abundance of MPs near urban centers and large lakes that are part of the Yangtze River system, and in the river estuary, indicate that both human activity and large bodies of open water affect MPs density in shore and bank sediment. Flake particles were the major constituents in all upstream samples, and foam particles were predominant in downstream samples. The distribution of foam particles displayed an east-west pattern of stepwise descent, but flake and film particles showed the opposite distribution. Small MP particles $(<0.050 \mathrm{~mm})$ were predominant at all sites in this study; the minimum size of particles in the Yangtze River banks was $0.065 \mathrm{~mm}$. There was a significant correlation between MP particle abundance and mean annual runoff of the tributary basin in the upper Yangtze river tributaries. There was an order of magnitude reduction in average abundance of MP particles in lakeshore sediments between lake inlet and outlet due to particle interception associated with sedimentation and precipitation in the lake. Systematic surveys of hydro-sedimentary parameters and better characterization of primary plastics sources are needed to better understand the hydrodynamic drivers of the distribution and persistence of plastic particles in the Yangtze River. The MP particle abundances in sediments can act as a proxy for the spatial extension of human activities that are propagated across the Yangtze River basin, and they are also a fingerprint of pollution sources.

\section{Declarations}

Author contribution All authors contributed to the study conception and design. Material preparation, data collection, and analysis were performed by Wenfeng Li, Jia Duo, Rehemanjiang Wufuer, and Shuzhi Wang. The first draft of the manuscript was written by Wenfeng Li. Professor Xiangliang Pan supervised the research and did critical revision of the manuscript. All authors have contributed considerably, and all authors are in agreement with respect to the manuscript content. The authors read and confirmed the final manuscript.

Funding The authors gratefully acknowledge the financial support by the National Natural Science Foundation of China (No. 41907140), the Natural Science Foundation of Xinjiang Uygur Autonomous Region (No. 2018D01A38) and the West Light Foundation of Chinese Academy of Sciences (No. 2018XBQNXZ-B-014; 2017-XBQNXZ-B-011), Science and Technology Department of Xinjiang Uyghur Autonomous Region (2018D04013) and High-level Talents Introduction Project in Xinjiang Uygur Autonomous Region (2018).

Availability of data and materials All data generated or analysed during this study are included in this published article (and its supplementary information files). 


\section{Compliance with ethical standards}

Ethics approval and consent to participate Not applicable.

Consent for publication Not applicable.

Competing interests The authors declare no competing interests.

\section{References}

1. Canesi L, Ciacci C, Bergami E, Monopoli MP, Dawson KA, Papa S et al. (2015) Evidence for immunomodulation and apoptotic processes induced by cationic polystyrene nanoparticles in the hemocytes of the marine bivalve Mytilus. Mar Environ Res 111: 34-40. https://doi.org/10.1016/j.marenvres.2015.06.008

2. Dai ML, Zhang MB (2013) Research on temporal and spatial distribution law of runoff in Yangtze River Basin. Yangtze River 44(10): 88-91.

3. Desforges JPW, Galbraith M, Dangerfield N, Ross PS (2014) Widespread distribution of microplastics in subsurface seawater in the NE Pacific Ocean. Mar Pollut Bull 79(1): 94-99. https://doi.org/10.1016/j.marpolbul.2013.12.035

4. Ding L, Mao RF, Guo XT, Yang XM, Zhang Q, Yang C (2019) Microplastics in surface waters and sediments of the Wei River, in the northwest of China. Sci Total Environ 667: 427-434. https://doi.org/10.1016/j.scitotenv.2019.02.332

5. Dong YH, Wang XL (2013) Preliminary Research on Watershed Division and Stream Order Classification of the Yangtze River. Journal of Yangtze River Scientific Research Institute. 230: 1-5.

6. do Sul JAl, Costa MF (2014) The present and future of microplastic pollution in the marine environment. Environ Pollut 185: 352-364. https://doi.org/10.1016/j.envpol.2013.10.036

7. Endo S, Takizawa R, Okuda K, Takada H, Chiba K, Kanehiro H et al. (2005) Concentration of polychlorinated biphenyls (PCBs) in beached resin pellets: Variability among individual particles and regional differences. Mar Pollut Bull 50(10): 1103-1114.

https://doi.org/10.1016/j.marpolbul.2005.04.030

8. Eriksen M, Mason S, Wilson S, Box C, Zellers A, Edwards W, et al. (2013) Microplastic pollution in the surface waters of the Laurentian Great Lakes. Mar pollut bull 77(1):177-82.

https://doi.org/10.1016/j.marpolbul.2013.10.007

9. Feng S., Lu HW, TC, Liu YL, Tian PP, Lu JZ (2021) Microplastic footprints in the Qinghai-Tibet Plateau and their implications to the Yangtze River Basin. J Hazard Mater 407: 124776-124785. https://doi.org/10.1016/j.jhazmat.2020.124776

10. Free CM, Jensen OP, Mason SA, Eriksen M, Williamson NJ, Boldgiv B (2014) High-levels of microplastic pollution in a large, remote, mountain lake. Mar Pollut Bull 85(1): 156-163. https://doi.org/10.1016/j.marpolbul.2014.06.001 
11. Gasperi J, Dris R, Bonin T, Roche V, Tassin B (2014) Assessment of floating plastic debris in surface water along the Seine River. Environl Pollut 195: 163-166.

https://doi.org/10.1016/j.envpol.2014.09.001

12. He D, Chen XJ, Zhao W, Zhu ZQ, Qi XJ, Zhou LF, Chen W, Wan CY, Li D., Zou X, Wu N (2021) Microplastics contamination in the surface water of the Yangtze River from upstream to estuary based on different sampling methods. Environ Res 196: 110908-110917. https://doi.org/10.1016/j.envres.2021.110908

13. Hidalgo-Ruz V, Gutow L, Thompson RC, Thiel M (2012) Microplastics in the Marine Environment: A Review of the Methods Used for Identification and Quantification. Environ Sci Tech 46(6): 3060-3075. https://doi.org/10.1021/es2031505

14. Hu L, Chernick M, Hinton DE, Shi H (2018) Microplastics in Small Waterbodies and Tadpoles from Yangtze River Delta, China. Environ Sci Techol 52(15): 8885-8893. https://doi.org/10.1021/acs.est.8b02279

15. Ivar do Sul JA, Costa MF (2014) The present and future of microplastic pollution in the marine environment. Environ Pollut 185: 352-364. https://doi.org/10.1016/j.envpol.2013.10.036

16. Jambeck JR, Geyer R, Wilcox C, Siegler TR, Perryman M, Andrady A et al. (2015) Plastic waste inputs from land into the ocean. Science 347(6223): 768-771. https://doi.org/ 10.1126/science.1260352

17. Jian MF, Zhang Y, Yang WJ, Zhou LY, Liu SL 2020. Occurrence and distribution of microplastics in China's largest freshwater lake system. Chemosphere, 216: 128186-128195. https://doi.org/10.1016/j.chemosphere.2020.128186

18. Klein S, Worch E, Knepper TP (2015) Occurrence and Spatial Distribution of Microplastics in River Shore Sediments of the Rhine-Main Area in Germany. Environ Sci Technol 49(10): 6070-6076. https://doi.org/10.1021/acs.est.5b00492

19. Lahens L, Strady E, Kieu-Le TC, Dris R, Boukerma K, Rinnert E, Gasperi J, Tassin B(2018) Macroplastic and microplastic contamination assessment of a tropical river (Saigon River, Vietnam) transversed by a developing megacity. Environ Pollut 236: 661-671. https://doi.org/10.1016/j.envpol.2018.02.005

20. Lebreton LCM, Van der Zwet J, Damsteeg JW, Slat B, Andrady A, Reisser J ( 2017) River plastic emissions to the world's oceans. Nat Commun 8. https://doi.org/10.1038/ncomms 15611

21. Lee J, Lee JS, Jang YC, Hong SY, Shim WJ, Song YK et al. (2015) Distribution and Size Relationships of Plastic Marine Debris on Beaches in South Korea. Arch Environ Con Tox 69(3): 288-298. https://doi.org/10.1007/s00244-015-0208-x

22. Li J, Liu H, Paul Chen J (2018) Microplastics in freshwater systems: A review on occurrence, environmental effects, and methods for microplastics detection. Water Res 137: 362-374. https://doi.org/10.1016/j.scitotenv.2020.141948

23. Lin L, Zuo LZ, Peng JP, Cai LQ, Fok L, Yan Y, Li HX, Xu XR (2018) Occurrence and distribution of microplastics in an urban river: a case study in the Pearl River along Guangzhou City, China. Sci Total Environ 644: 375-381. https://doi.org/10.1016/j.scitotenv.2018.06.327 
24. Liu C, Li J, Zhang Y, Wang L, Deng J, Gao Y, et al. (2019) Widespread distribution of PET and PC microplastics in dust in urban China and their estimated human exposure. Environ Int 128:116-24. https://doi.org/10.1016/j.envint.2019.04.024

25. Mathalon A, Hill P (2014) Microplastic fibers in the intertidal ecosystem surrounding Halifax harbor, Nova Scotia. Mar Pollut Bull 81: 69-79. https://doi.org/10.1016/j.marpolbul.2014.02.018

26. Murray F, Cowie P. (2011) Plastic contamination in the decapod crustacean Nephrops norvegicus (Linnaeus, 1758). Mar Pollut Bull 62(6): 1207-1217.

https://doi.org/10.1016/j.marpolbul.2011.03.032

27. Nguyen B, Claveau-Mallet D, Hernandez LM, Xu EG, Farner JM, Tufenkji N (2019) Speparation and analysis of microplasits and nanoplastics in complex environmental samples. Acc Chem Rews 52 : 858-866. https://doi.org/10.1021/acs.accounts.8b00602

28. Nor NHM., Obbard JP (2014) Microplastics in Singapore's coastal mangrove ecosystems. Mar Pollut Bull 79 (1): 278-283. https://doi.org/10.1016/j.marpolbul.2013.11.025

29. Peng G, Xu P, Zhu B, Bai M, Li D (2018) Microplastics in freshwater river sediments in Shanghai, China: A case study of risk assessment in mega-cities. Environ Pollut 234: 448-456. https://doi.org/10.1016/j.envpol.2017.11.034

30. Plastics Europe (2018) Plastics-the Facts 2018, an Analysis of European Plastics Production, Demand and Waste Data. https://www.plasticseurope.org/en/resources/ market-data, Accessed date: 22 March 2019. Plastics Europe.

31. Rachman CM (2018) Microplastics research-from sink to source. Science 360, 28-29. https://doi.org/10.1126/science.aar7734

32. Rech S, Macaya-Caquilpán V, Pantoja JF, Rivadeneira MM, Jofre Madariaga D, Thiel M (2014) Rivers as a source of marine litter- A study from the SE Pacific. Mar Pollut Bull 82(1): 66-75. https://doi.org/10.1016/j.marpolbul.2014.03.019

33. Ryan Peter G, Moore Charles J, van Franeker Jan A, Moloney Coleen . (2009) Monitoring the abundance of plastic debris in the marine environment. Series B, Biological sciences 364(1526): 1999-2012. https://doi.org/10.1098/rstb.2008.0207

34. Siegfried M, Koelmans AA, Besseling E, Kroeze C (2017) Export of microplastics from land to sea. A modelling approach. Water Res 127: 249-257. https://doi.org/10.1016/j.watres.2017.10.011

35. Sintim HY. Flury M (2017) Is Biodegradable Plastic Mulch the Solution to Agriculture's Plastic Problem? Environ Sci Technol 51(3): 1068-1069. https://doi.org/10.1021/acs.est.6b06042

36. Song YK, Hong SH, Jang M, Han GM, Jung SW, Shim WJ (2017) Combined effects of UV exposure duration and mechanical abrasion on microplastic fragmentation by polymer type. Environ Sci Technol 51: 4368-4376. https://doi.org/10.1021/acs.est.8b00172

37. Su L, Xue Y, Li L, Yang D, Kolandhasamy P, Li D et al. (2016) Microplastics in Taihu Lake, China. Environ Pollut 216: 711-719. https://doi.org/10.1016/j.envpol.2016.06.036

38. Turner A, Holmes L (2011) Occurrence, distribution and characteristics of beached plastic production pellets on the island of Malta (central Mediterranean). Ma Pollut Bull 62(2): 377-381. 
https://doi.org/10.1016/j.marpolbul.2010.09.027

39. Wang W, Ndungu AW, Li Z, Wang J (2017) Microplastics pollution in inland freshwaters of China: a case study in urban surface waters of Wuhan, China. Sci Total Environ 575: 1369-1374. https://doi.org/10.1016/j.scitotenv.2016.09.213

40. Wang W, Yuan W, Chen Y, Wang J (2018) Microplastics in surface waters of Dongting Lake and Hong Lake, China. Sci Total Environ 633: 539-545. https://doi.org/10.1016/j.scitotenv.2018.03.211

41. Woodall Lucy C, Sanchez-Vidal A, Canals M, Paterson Gordon LJ, Coppock R, Sleight V et al. (2014) The deep sea is a major sink for microplastic debris. Roy Soc Open Sci (4): 140317. https://doi.org/10.1098/rsos.140317

42. Wu C, Zhang K, Xiong X (2018) Microplastic pollution in inland waters focusing on Asia. Spring Chem 58: 85-99.

43. Xiong X, Wu C, Elser J., Mei Z, Hao Y (2019) Occurrence and fate of microplastic debris in middle and lower reaches of the Yangtze River-From inland to the sea. Sci Total Environ 659: 66-73. https://doi.org/10.1016/j.scitotenv.2018.12.313

44. Xu P, Peng G, Su L, Gao Y, Gao L, Li D (2018) Microplastic risk assessment in surface waters: A case study in the Changjiang Estuary, China. Mar Pollut Bull 133: 647-654. https://doi.org/10.1016/j.marpolbul.2018.06.020

45. Yang L, Zhang YL, Kang S, Wang ZQ, Wu CX (2021) Microplastics in freshwater sediment: A review on methods, occurrence, and sources. Sci Total Environ 754: 141948-141968. https://doi.org/10.1016/j.scitotenv.2020.141948

46. Yao L, Chen, PP, Hu LL, Li YQ (2016) Research on the Status and Problems of the Upper Yangtze River Hydropower Development. Journal of Mianyang Teachers'College. 35: 91-97.

47. Yonkos L., Friedel EA, Perez-Reyes A., Ghosal S, Arthur CD (2014) Microplastics in Four Estuarine Rivers in the Chesapeake Bay, U.S.A. Environ Sci Technol 48(24):14195-202. https://doi.org/10.1021/es5036317

48. Zhao S, Zhu L, Wang T, Li D (2014) Suspended microplastics in the surface water of the Yangtze Estuary System, China: First observations on occurrence, distribution. Mar Pollut Bul 86(1): 562-568. https://doi.org/10.1016/j.marpolbul.2014.06.032

49. Zhao S, Zhu L, Li D (2015) Microplastic in three urban estuaries, China. Environ Pollut 206: 597-604. https://doi.org/10.1016/j.envpol.2015.08.027

50. Zhang H, Zhou Q, Xie Z, Zhou Y, Tu C, Fu C et al. (2018) Occurrences of organophosphorus esters and phthalates in the microplastics from the coastal beaches in north China. Sci Total Environ 616617: 1505-1512. https://doi.org/10.1016/j.scitotenv.2017.10.163

51. Zhang K, Gong W, Lv J, Xiong X, Wu C (2015) Accumulation of floating microplastics behind the Three Gorges Dam. Environ Pollut 204: 117-123. https://doi.org/10.1016/j.envpol.2015.04.023

52. Zhang K, Xiong X, Hu HJ, Wu CX, Bi YH, Wu YH, Zhou BS, Lam PKS, Liu JT (2017) Occurrence and characteristics of microplastic pollution in Xiangxi Bay of Three Gorges Reservoir, China. Environ Sci Technol 51: 3794-3801. https://doi.org/10.1021/acs.est.7b00369 
53. Zhou Q, Zhang H, Fu C, ZhouY, Dai Z, Li Y et al. (2018) The distribution and morphology of microplastics in coastal soils adjacent to the Bohai Sea and the Yellow Sea. Geoderma 322: 201-208. https://doi.org/10.1016/j.geoderma.2018.02.015

54. Zhou Q, Zhang HB, Zhou Y, Li Y, Xi, Y, Fu C., Tu C, Luo YM (2016) Separation of microplastics from a coastal soil and their surface microscopic features. Chin Sci Bull 61: 1604-1611. https://doi.org/10.1360/N972015-01098

\section{Figures}

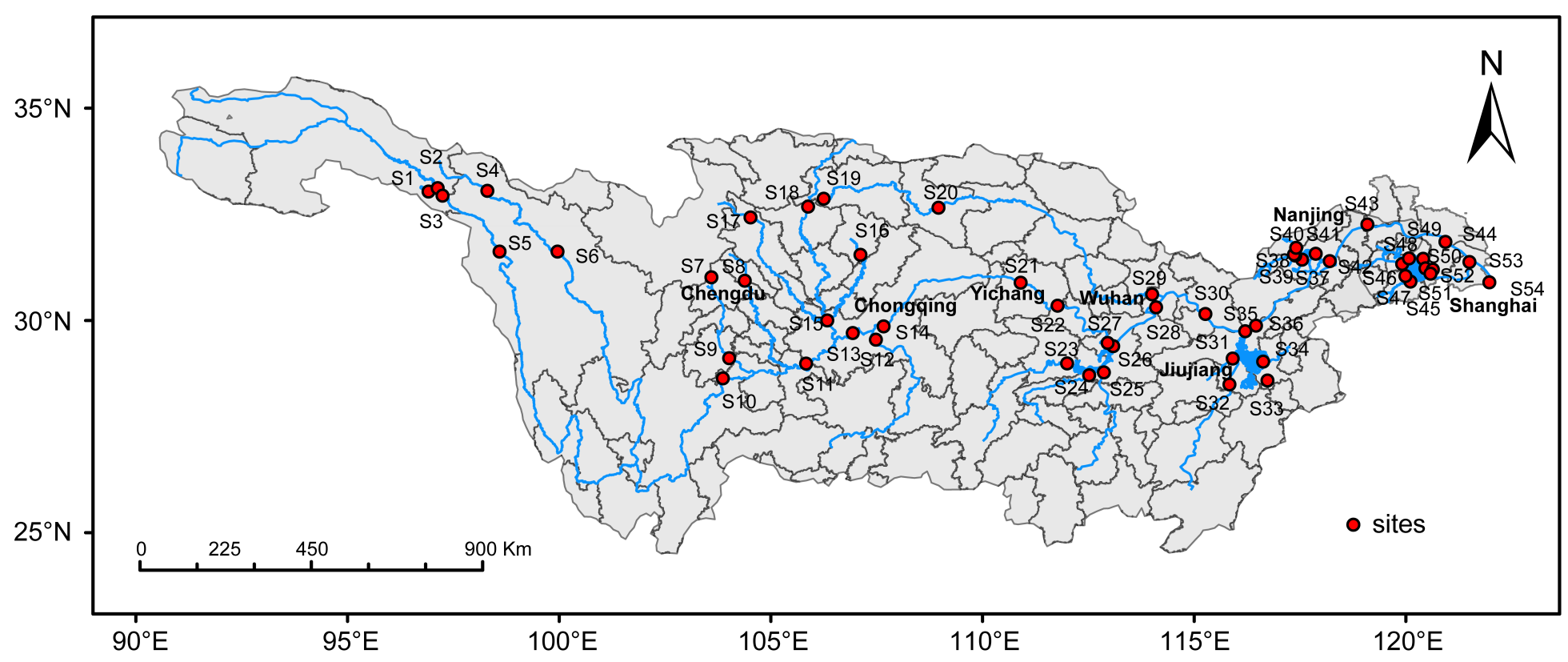

Figure 1

Sketch map showing the sampling sites along the Yangtze River valley. Drawing number: GS(2019)1653. Note: The designations employed and the presentation of the material on this map do not imply the expression of any opinion whatsoever on the part of Research Square concerning the legal status of any country, territory, city or area or of its authorities, or concerning the delimitation of its frontiers or boundaries. This map has been provided by the authors. 

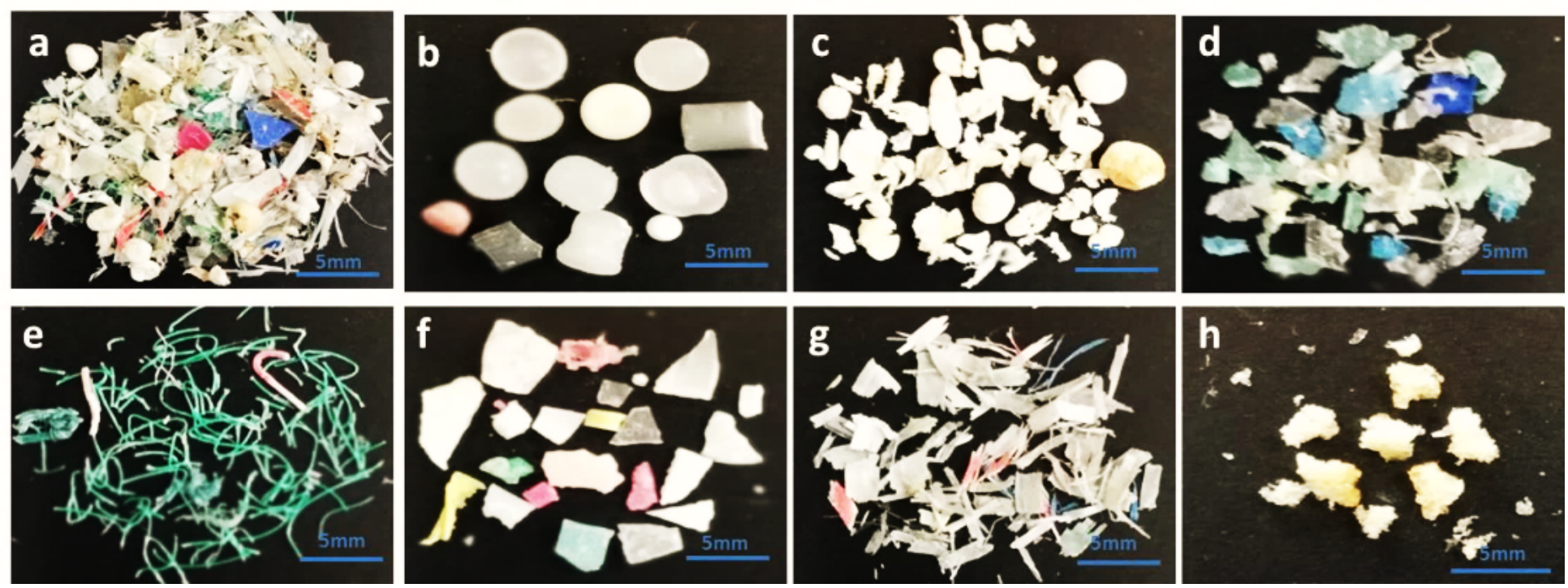

Figure 2

Different shape categories of partial microplastic samples collected from the shoreside sediments along the Yangtze River; a. mixed MPs, b. pellets, c. foams, d. films, e. fibers, f. fragments, g. flakes, h. sponges.

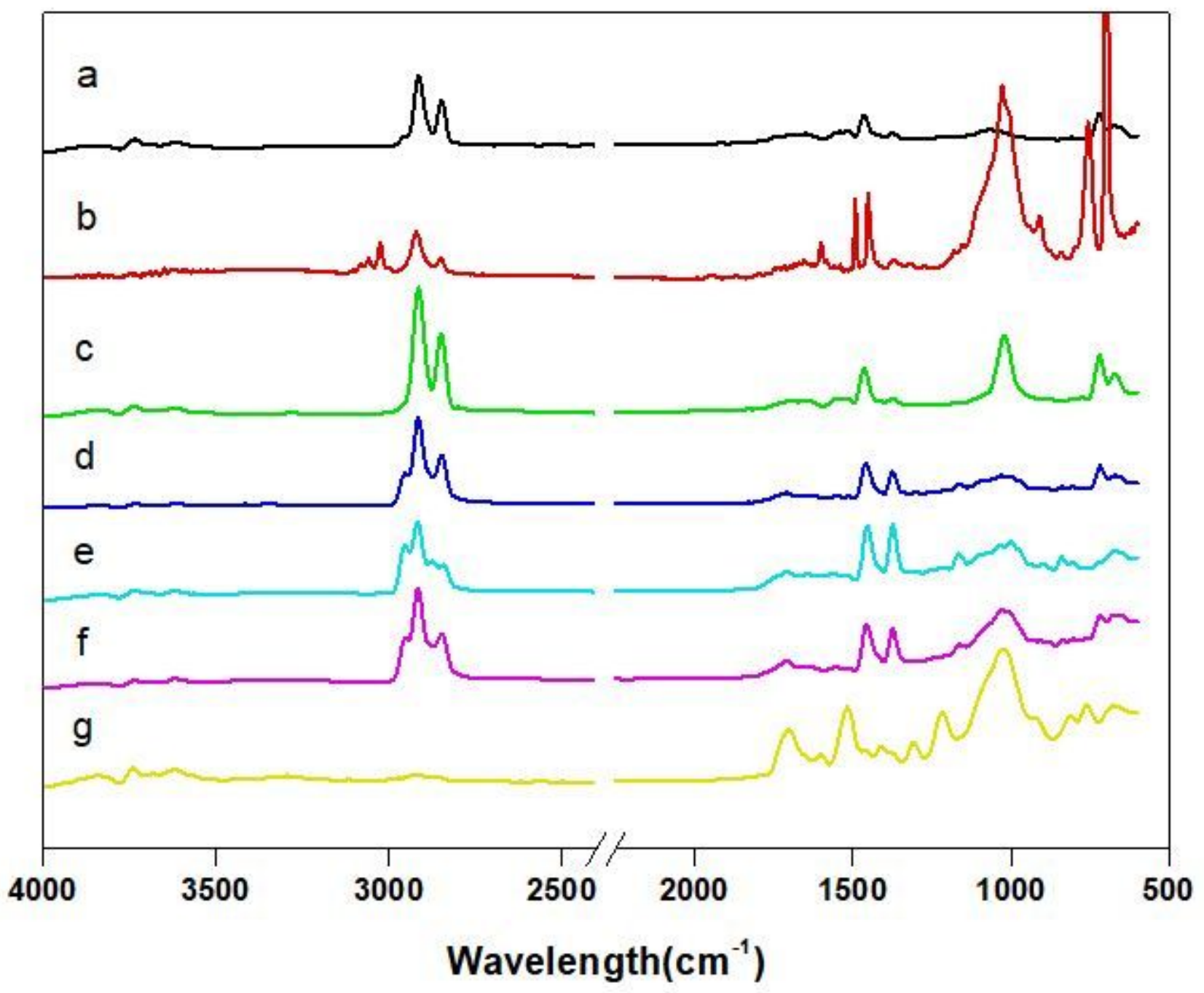


Figure 3

ATR-FTIR spectras of different microplastic samples from the Yangtze river valley; a. pellet, b. foam, c. film, d. fiber, e. fragment, f. flake, g. sponge.
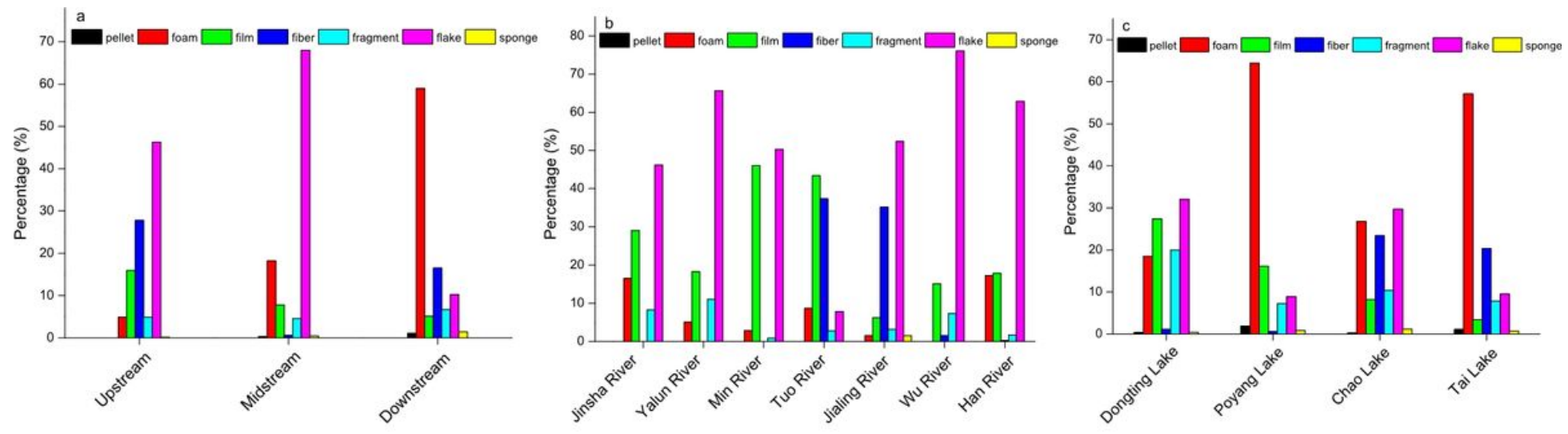

Figure 4

Shape categories of MPs in sediments along the Yangtze River, (a) Upper, middle and lower reaches of the Yangtze River; (b tributaries; (c) lakes.
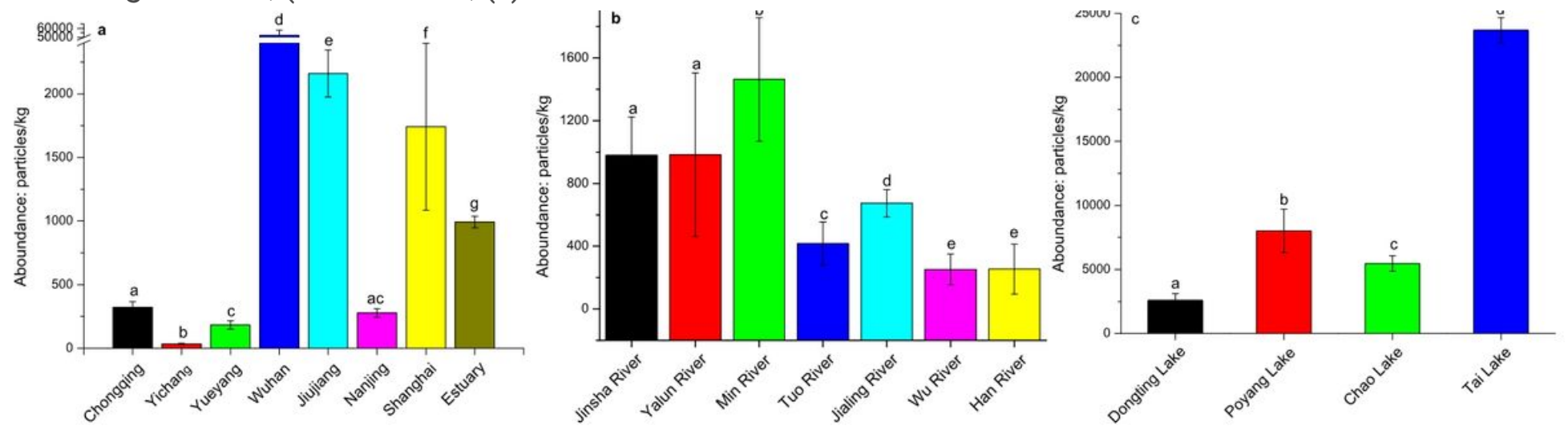

\section{Figure 5}

MPs abundance in sediments along the Yangtze River,Upper, middle and lower reaches of the Yangtze River $(a, n=3)$; Tributaries ( $b, n=3)$; Lakes $(c, n=3)$. Different letters above the bars indicate significant differences $(P<0.05)$, while same letters indicate no significant difference $(P>0.05)$. 

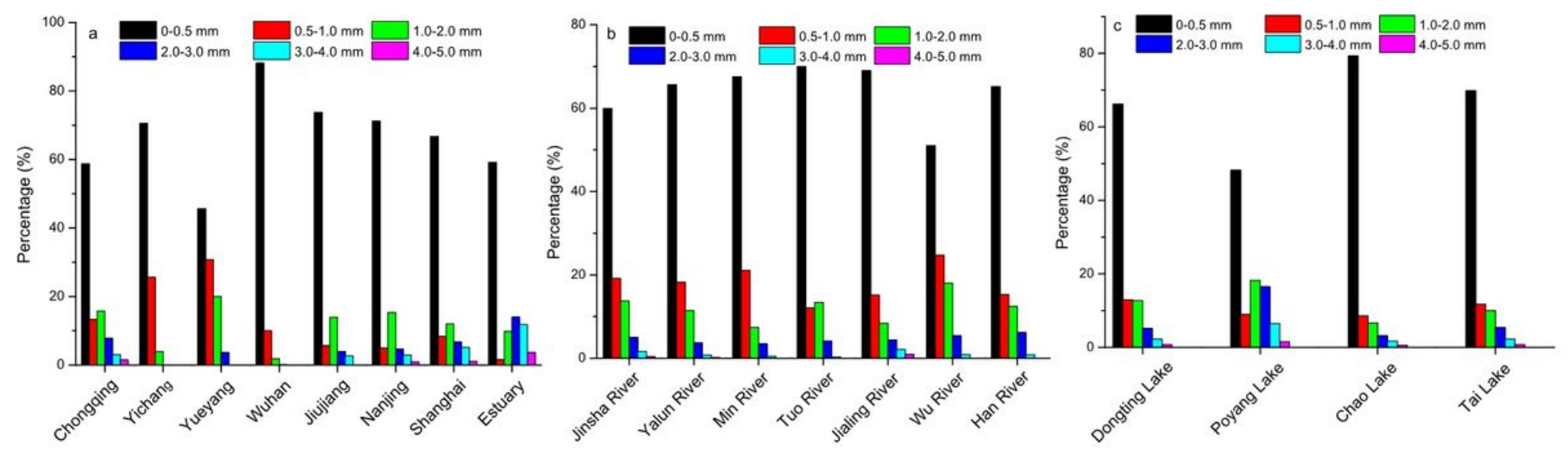

Figure 6

Size distribution of MPs in shoreside sediments; (a) Upper, middle and lower reaches of the Yangtze River;

(b) tributaries; (c) lakes.
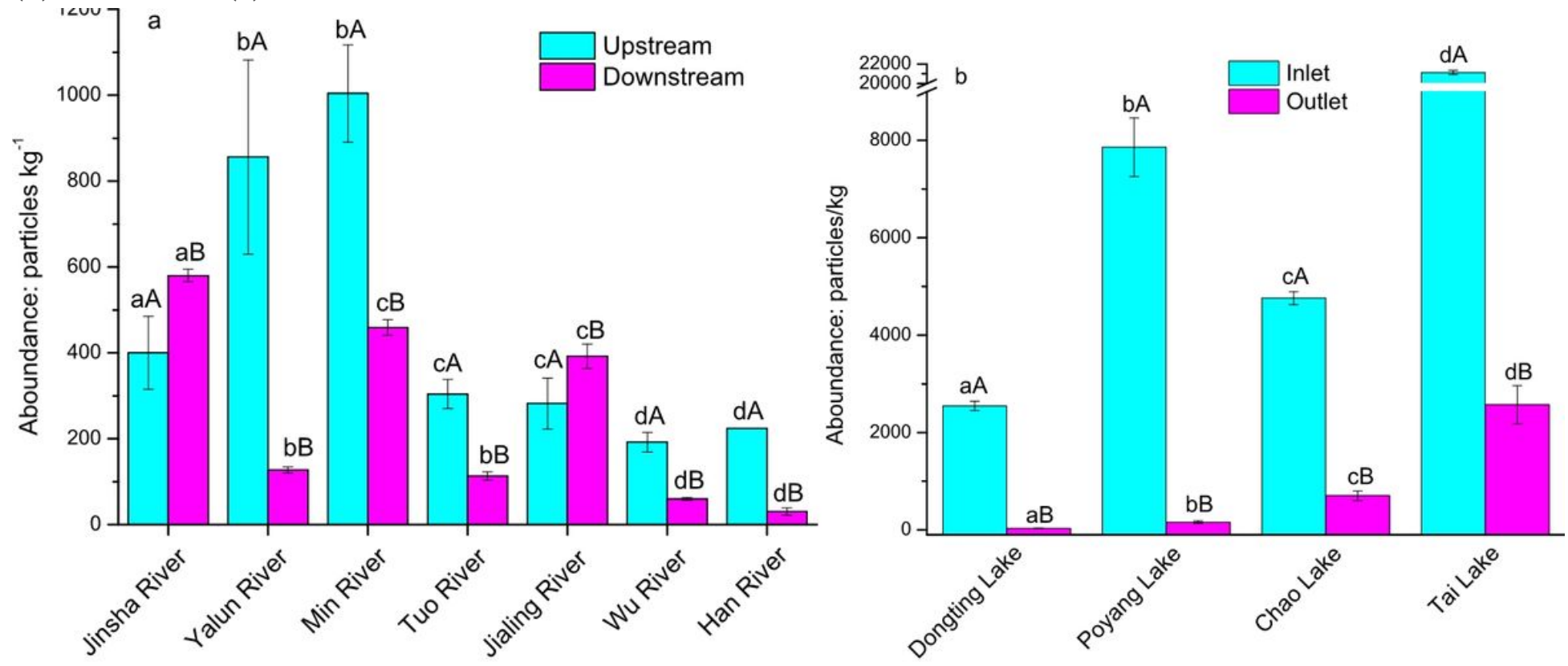

Figure 7

Microplastic abundance in the sediments of each tributary upstream and downstream (a) and the inlet and outlet of lakes (b). One-way analysis of variance (ANOVA) with Dunnett T3 post-hoc test was performed to determine differences in particle number among different treatment groups $(a, b, n=3)$. The letters above the bars indicate significant differences $(p<0.05)$. Different capital letters represent significant differences between upstream and downstream of the same tributary / inlet and outlet of the same lake, and different lowercase letters represent significant differences between different tributaries / lakes at the same site. 

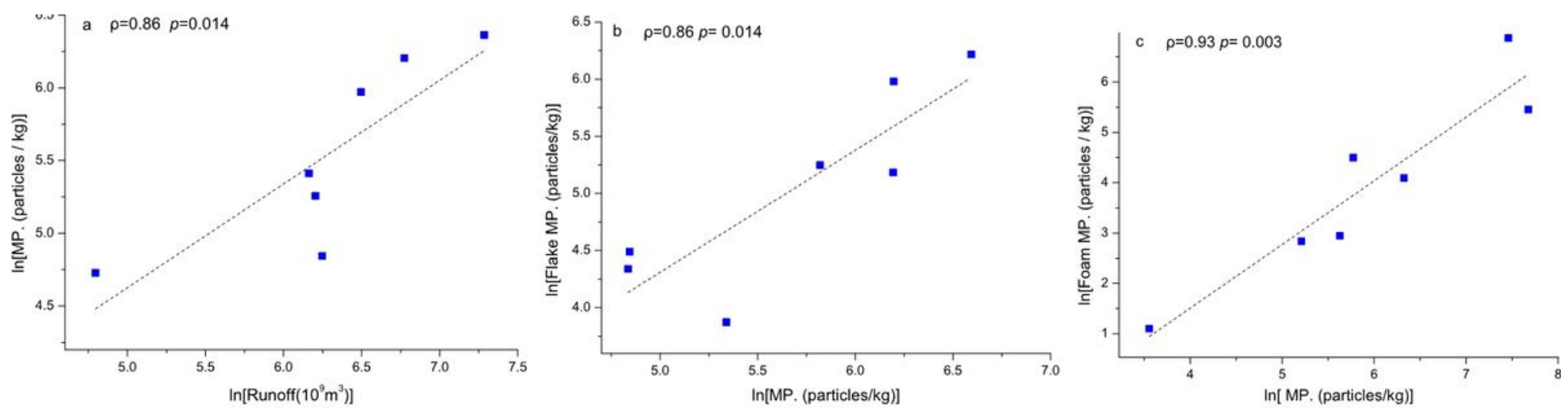

\section{Figure 8}

Significant correlations of MPs abundance and other parameters. (a) MPs of abundance and mean annual runoff in the main tributary; (b) abundance of flake MPs and total MPs in the main tributary; (c) abundance of foam MPs and total MPs in the main sampling areas of the Yangtze River.

\section{Supplementary Files}

This is a list of supplementary files associated with this preprint. Click to download.

- Graphicalabstract.jpg

- Supplementarylnformation.pdf 